Interfaces

\title{
La litre, ceinture de deuil ou trait ultime
}

Jeanette Zwingenberger

\section{(2) OpenEdition}

Journals

Édition électronique

URL : http://journals.openedition.org/interfaces/870

DOI : 10.4000/interfaces.870

ISSN : 2647-6754

Éditeur :

Université de Bourgogne, Université de Paris, College of the Holy Cross

Édition imprimée

Date de publication : 15 juillet 2020

Pagination : 51-64

ISSN : 1164-6225

\section{Référence électronique}

Jeanette Zwingenberger, « La litre, ceinture de deuil ou trait ultime », Interfaces [En ligne], 43 | 2020, mis en ligne le 15 juillet 2020, consulté le 07 janvier 2021. URL : http://journals.openedition.org/ interfaces/870 ; DOI : https://doi.org/10.4000/interfaces.870

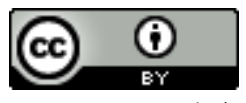

Les contenus de la revue Interfaces sont mis à disposition selon les termes de la Licence Creative Commons Attribution 4.0 International. 


\title{
LA LITRE, CEINTURE DE DEUIL OU TRAIT ULTIME
}

\author{
Jeanette Zwingenberger \\ PhD Université Paris 1 Panthéon-Sorbonne, commissaire d'exposition indépendante, \\ membre de l'AICA (International Association of Art Critics) et membre suppléant du \\ Comité consultatif pour les ouvres d'art de l'UNESCO
}

\begin{abstract}
Résumé : Un bandeau noir peint sur le pourtour des murs de l'église et sur lequel figurent les armoiries seigneuriales, appelé litre, révèle un dispositif funéraire. Ce rite fut aboli avec la Révolution, effacé et oublié, il constitue aujourd'hui un enjeu crucial pour la restauration, les monuments historiques, bref notre mémoire. Nous analysons ici l'histoire du droit de litre en relation à l'espace sacré dans sa dimension politique, sociale et anthropologique. Le noir en tant que couleur de deuil renvoie à l'irreprésentable. Trait ultime, il annonce justement l'espace de l'abstraction.

Mots-clés : : anthropologie des image, art et histoire, représenter le deuil, restauration, vie et mort des images, trait ultime de l'abstraction

Abstract: A black band painted on the circumference of the walls of the church and on which appear the seigniorial coat of arms, called litre, reveals a funeral custom. This rite was abolished with the Revolution, erased and forgotten, it is today a crucial issue for restoration, historical monuments and our memory. We analyze the history of the right of litre in relation to the sacred space in its political, social and anthropological dimension. Black as a mourning color refers to the irrepresentable; as an ultimate line, it announces the space of abstraction.
\end{abstract}

Keywords: anthropology of images, art and history, representing mourning, restauration, life and death of images, ultimate line of abstraction 


\section{Hommage à Solange Fouilleul}

« La mort, - si nous voulons nommer ainsi cette irréalité - est ce qu'il y a de plus terrible et maintenir l'œuvre de la mort est ce qui demande la plus grande force [...] Qu'il vive ou qu'il meure, l'homme ne peut connaître immédiatement la mort. La connaissance de la mort ne peut se passer d'un subterfuge : le spectacle ».

(Hegel 29)

De la crainte de la mort naît l'art. La force de la peinture et surtout du portrait depuis l'époque de Fayoum réside dans son pouvoir de présentifier le défunt qui reprend vie dans le regard du spectateur. La personne survit grâce à son portrait qui a une valeur de substitution. La coutume de la ceinture funéraire, par contre, inverse cette logique en faisant porter le deuil à l'espace sacré et parfois aux images qu'elle traverse.

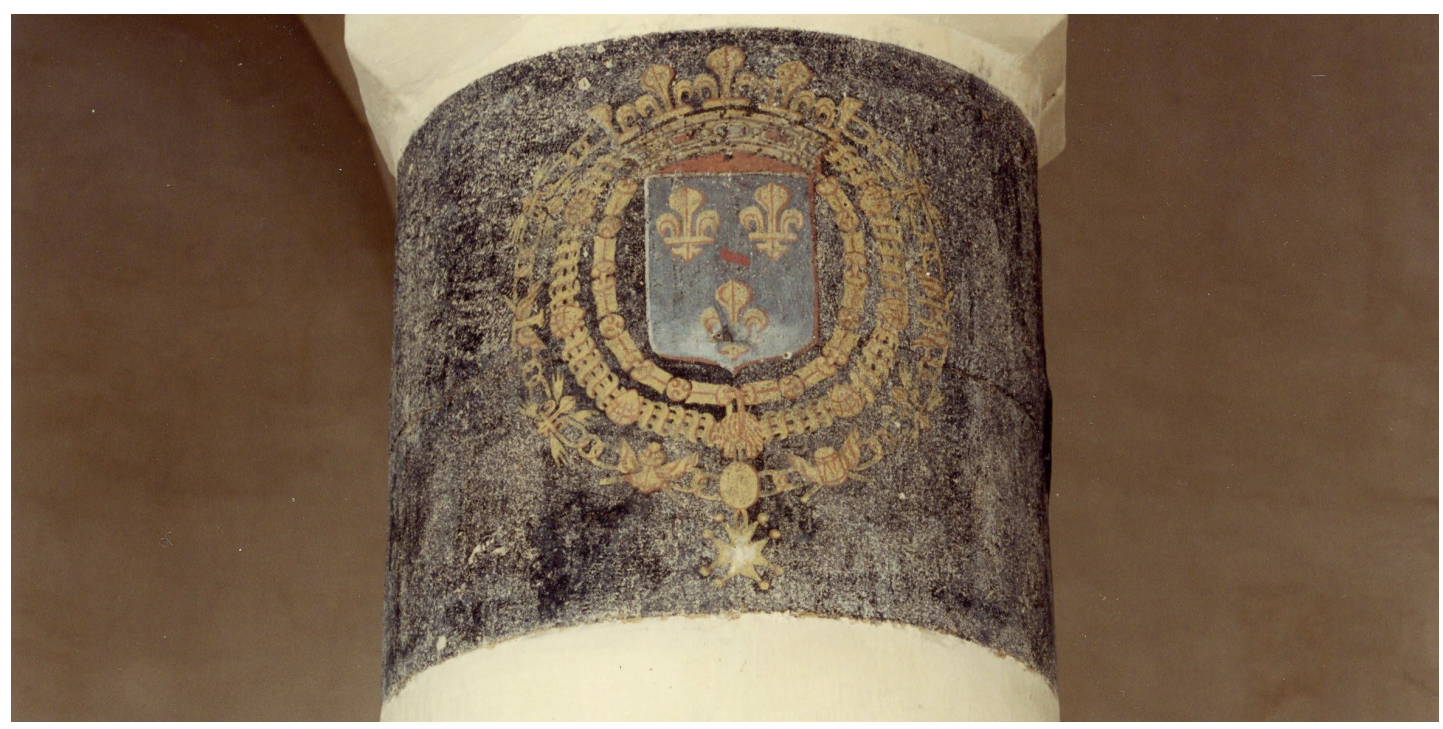

Fig. 1. Un exemple significatif est cette litre funéraire de l'église de Courteuil (Oise) avec les armoiries du Grand Condé, probablement du XVIII ${ }^{\mathrm{èm}}$ siècle. Photographie Gilles Gaultier 
La litre est un dispositif funéraire consistant en un bandeau noir (composé d'un mélange de noir de fumée et de colle de peau) peint sur le pourtour des murs de l'église et sur lequel figurent les armoiries seigneuriales. Il rappelle la coutume voulant qu'une bande noire soit tendue sur les murs de l'église à l'occasion des funérailles (Bodin 239). Une des premières litres peintes revient à Colard de Laon en I397. Le rôle de cette ceinture est de perpétuer la mémoire du défunt le temps du deuil, durant un an et un jour. Cette tradition, abolie avec l'Ancien Régime, faisait partie des droits honorifiques réservés à certains seigneurs patrons d'église, bienfaiteurs. La coutume consistant à arborer un brassard noir en signe de deuil en témoigne jusqu'à aujourd'hui. Que nous apprend la couleur noire dans son lien avec le deuil et les armoiries par rapport à la personne défunte qu'elle représente ?

Nous n'aborderons pas le vaste sujet des décors funéraires, nous nous limiterons ici à la litre et à son fonctionnement dans une perspective anthropologique. Le mot litre renvoie au mot latin lino qui se rattache à litura et signifie « rature, correction » et « tache », mais aussi linum, fil à coudre, corde. Linteum : étoffe de lin, linge et voile (Ernout et Meillet 360-6I). Il remonte au latin médiéval listra et ses variantes lictra, litra la « bordure ». Cette lisière est le bord extrême, la frange que referme la mort. Mors ultima linea rerum est, écrit Horace ${ }^{1}$ (Épîtres, livre I, I6, vers 79). Il se réfère à la ligne d'arrivée des courses dans les arènes de l'époque romaine et au fil du destin.

\section{Les enjeux de leur conservation in situ}

Nous analyserons ici le fonctionnement de la litre. Ce dispositif funéraire est lié à une personne et à une architecture sacrée. Le bandeau noir brise la blancheur des murs en interrompant l'esthétique de la chapelle. Souvent ne subsistent de ce décor éphémère que des « taches » noires. La litre est parfois placée en-dessous ou au-dessus d'une peinture murale, elle peut également la traverser. La taille de la litre peut varier de $40 \mathrm{~cm}$ à I,20m de hauteur ${ }^{2}$. Deux à trois litres peuvent traverser l'intérieur de l'église, comme c'est le cas à Vanlay (Fig. 2).

« La mort est la dernière limite des choses ». Traduction de l'auteure.

Selon Gilles Gaultier, qui est restaurateur de peintures murales à Paris. 


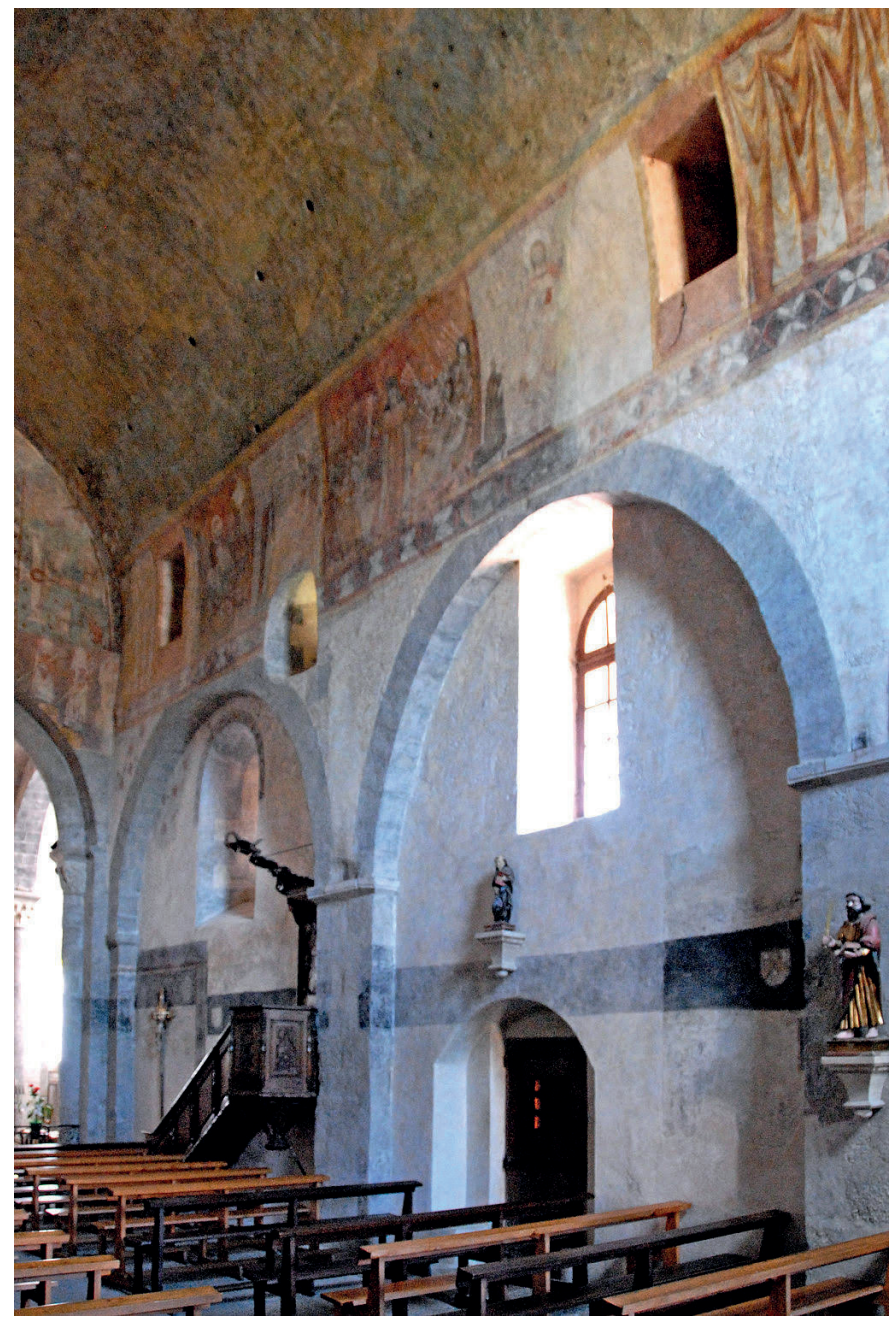

Fig. 2. L'église de Vanlay, département de l'Aube. Photographie Ilona Hans Colas 
Comment rendre visible et garder la mémoire de ces pratiques éphémères ? Il y a là aujourd'hui un enjeu central pour la restauration des églises. L'étude stratigraphique du décor permet d'identifier les couches picturales successives, réalisées à des époques différentes : décors figurés, litres, armoiries, couches de badigeon. Cette superposition de couches de différents médiums et de sujets traduit les transformations successives du décor pictural dans une église, mais aussi la vie et la mort des images. Souvent l'arrivée d'un nouveau seigneur patron inaugurait un nouveau cycle de peinture. L'enjeu crucial pour les restaurateurs aujourd'hui est donc de savoir quel sujet et quelle couche temporelle seront mis au jour, dégagés et préservés.

Selon Laurence Blondaux, les litres conservées à l'extérieur des églises sont de plus en plus rares. Bien souvent, leur mauvais état de conservation incite à les supprimer. Elles ne sont conservées que si elles portent des armoiries, dont nous avons aujourd'hui souvent perdu la signification. Les litres n'existaient que le temps du deuil, elles sont donc par nature éphémères. Elles étaient ensuite recouvertes d'un badigeon blanc effaçant ainsi le décor pictural. Laurence Blondaux évoque un exemple en Côte d'Or, dans l'église Saint-Pierre-aux-Liens de Bâlot, où les peintures murales ont été restaurées en 2004 (Blondaux 319-24). Au centre du mur ouest, à l'intérieur de la chapelle sud, la litre funéraire porte le blason de la famille de Sennevoy. Dans la chapelle nord, le blason du mur ouest, visible en transparence sous le badigeon, ne pouvait être dégagé sans risque de perte. En raison d'une interaction chimique, le badigeon s'est en effet coloré en brun au contact du pigment rouge sous-jacent. La forme du blason était donc visible mais le dégagement du badigeon difficile. La plupart du temps, les deux couches sont intimement mêlées en ces endroits si bien que l'on arrache souvent le pigment. La bande noire de la litre a été néanmoins restituée dans la chapelle nord. Dans la nef, sur le mur nord, de l'autre côté de la chaire, les mêmes armoiries timbrées et supportées par deux lions figurent sur un bandeau noir et ont été restaurées. Citons un autre exemple, dégagé en 2005 à l'église Saint-Martin de Mézières-en-Drouais (Eure-et-Loire) (Fig. 3) ${ }^{3}$. 


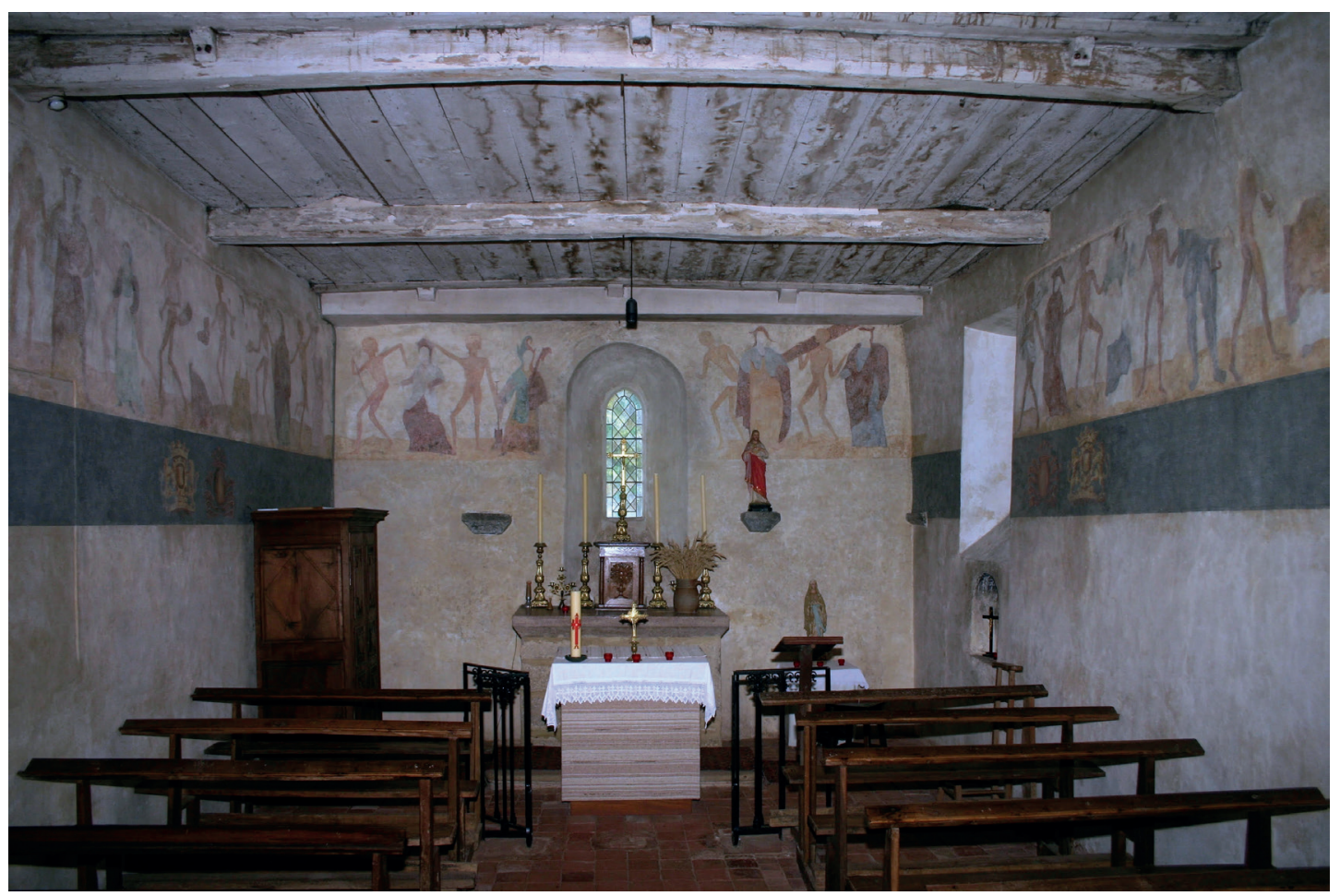

Fig. 3. Saint-Martin de Mézières-en-Drouais (Eure-et-Loire).

La Rencontre des trois morts et des trois vifs. Photographie Ilona Hans Colas

La Rencontre des trois morts et des trois vifs se trouve sur le mur nord (dimensions estimées de la partie visible : longueur 2,08 $\mathrm{m}$, hauteur 2,IO m, à I, $85 \mathrm{~m}$ du sol actuel) et semble pouvoir être datée de la première moitié du XVI ème siècle. Seuls les trois morts sont aujourd'hui visibles, ils partent du fond de l'église en direction des vifs. Sur le mur ouest, dans le prolongement de la Rencontre des trois morts et des trois vifs, on découvre deux couches superposées avec une litre funéraire passant sous la peinture figurative associée à une croix de consécration en couche monochrome, contemporaine. 
Les armoiries de la litre « d'argent à l'aigle de sable » correspondent à l'armorial Navarre aux armes d'Amfreville. On sait que de I455 à I552, la seigneurie de Mézières-sur-Eure était dans la famille d'Amfreville, avant de passer à la famille Balsac d'Entragues. La litre a donc certainement été réalisée durant cette période.

\section{L'histoire du droit de litre}

Christian Davy et Laurence Blondaux ont pu localiser le plus ancien énoncé relatif au droit de litre avec l'ordonnance de Villers-Cotterêts de I539. Par les articles I3 et I4, le roi François I ${ }^{\text {er }}$ étendait à toute la France la règle déjà valable pour la Bretagne, et stipulait que nul ne pouvait prétendre aux droits honorifiques s'il n'était patron ou fondateur (Davy et Blondeaux 3-I3). Le « droit de litre » était accordé aux patrons fondateurs des églises, les seigneurs féodaux. Leurs enfants, leurs successeurs pouvaient les faire peindre seulement à l'intérieur de l'église et non au-dehors (André). Ces grands propriétaires aumônaient les églises de leurs territoires et, en échange, le privilège du droit de litre leur était accordé. Alors que le patron avait droit de litre, dans le cas des nobles ou des officiers, les litres étaient faites d'une bande d'étoffe et d'écussons volants et étaient exposées dans l'église durant un an et un jour.

Plus tard, en I6I5, l'avocat au parlement de Paris, Mathias Maréchal décrit également, dans son traité, les droits honorifiques des seigneurs dans les églises et aborde spécifiquement la réglementation des litres dans l'espace sacré (Davy et Blondaux 3-I3). Le patron avait sa litre au-dessus de celle du haut-justicier à l'intérieur de l'église, en revanche à l'extérieur elle figurait au-dessous. Lorsqu'il y avait deux patrons, le seigneur suzerain avait le droit de faire poser sa litre au-dessus de celle de l'autre. On choisissait donc l'emplacement de sa litre de son vivant :

S'ils étaient [...] égaux, l'aîné mettait sa litre à droite et le puîné à gauche, ou bien celle de l'aîné était peinte au-dessus de celle du puîné. Dans le cas où la curie était divisée en deux, le patron de la première portion choisissait un côté du chœur et celui de la deuxième, l'autre côté en parallèle. Les femmes de condition libre et les veuves pouvaient faire apposer leur litre dans les églises dont elles avaient le patronage (Davy et Blondaux 3-13).

Au XVIII ${ }^{\text {me }}$ siècle, les armes des femmes étaient accolées à celles de leurs époux. Les patrons ecclésiastiques avaient également le droit de faire peindre leur litre mais seulement avec les armes de leur église. Le plus souvent ils n'en usaient point ou faisaient apposer une ceinture funèbre sans armoiries. 
Une des premières explications relatives à cette pratique est donnée en 1582 par le père Ménestrier : «Quoy qu'il n'y ait rien de si triste que les images de la mort, la vanité n'a pas laissé d'y introduire une espèce de luxe $[\ldots]$ ». Il parle ensuite des pompes funèbres : " Mon dessein est de traiter ces Décorations que l'usage a introduites, ou plutôt renouvelées depuis ces derniers siècles, lorsqu'on ajoute 'aux tentures noires', aux lumières et au chant lugubre de l'église, des inscriptions, des peintures et des représentations » (Ménestrier 274-82, 343-45). L'engouement pour les litres et leurs superpositions exprime la volonté de manifester ce temps de la mort. Cependant, dès le XVIII ${ }^{\text {ème }}$ siècle, la prolifération de la litre est règlementée par des arrêts du Parlement : « Le clergé n’appréciait que médiocrement ces privilèges qui portaient atteinte à son honneur sacerdotal. Par ailleurs il est évident que cette pratique avait tendance à défigurer les églises d'autant que certains seigneurs peu scrupuleux n'hésitaient pas à cette occasion à effacer non seulement les peintures mais encore les stations de chemin de croix et même les croix de consécration qui se trouvaient sur le trajet du bandeau funèbre » (Bodin 240).

L'évolution de la litre permet de conclure que l'espace sacré des murs et piliers des églises ou des chapelles initialement lié à l'enseignement religieux (chemin de croix, etc.) prend ici surtout une dimension politique et sociale qui lui fait perdre sa dimension religieuse. La Révolution y met d'ailleurs fin en interdisant l'usage des armoiries dans les lieux publics et fait ainsi disparaître ce privilège du droit de litre.

\section{Noir, couleur du deuil}

Madame de Saint-Simon meurt au 2/3 de la page II53 du manuscrit, le 2I janvier I743, date décidément tragique. Incapable de poursuivre la rédaction du grand œuvre, Saint-Simon dessine une ligne de larmes sur la page, avec, en leur centre, une croix - une ligne ou peut-être une litre, rappelant ces bandes de tissu noir semé d'emblèmes mortuaires avec lesquelles on drapait les maisons et les églises. Le trait est malhabile, le graphisme maladroit : quarante et une larmes de l'encre du malheur. [...] Saint-Simon ne reprendra son manuscrit que six mois plus tard (Raviez 48).

La couleur noire n'est associée à la mort qu'à partir du XIV ème siècle. La litre témoigne du changement de perception de cette couleur dorénavant associée aux trépassés. La première mention d'un deuil solennel en noir serait celui de la cour d'Angleterre à la mort de Jean II dit le Bon (I3I9I364). Auparavant on s'habillait de rouge, de vert, de bleu, de la couleur de ses plus beaux atours pour honorer le mort. 
La Peste amène dans son sillage le règlement vestimentaire en noir en tant qu'expression d'une véritable pénitence collective (Pastoureau 20I4, 95). Les premiers à annoncer ce changement de paradigme vestimentaire sont Philippe V de France en I3I6, à la mort de Louis X le Hutin, puis en I328, Mahaut d'Artois à la mort de Charles IV, son gendre. C'est surtout Philippe le Bon, le puissant duc de Bourgogne, qui impose à la cour de Bourgogne l'usage du noir. Le duc René II de Lorraine fit tendre de noir la collégiale Saint-Georges de Nancy pour célébrer les funérailles du duc de Bourgogne, Charles de Valois-Bourgogne, plus connu sous son surnom posthume de Charles le Téméraire (I433I477) (Pastoureau 20I4, 72). Anne de Bretagne est la première reine à se vêtir de noir à la mort de son époux Charles VIII (I498). Elle instaure ainsi la coutume des reines veuves, consistant à porter du noir en signe de deuil. À la mort de son second époux, Louis XII, elle se vêt de noir et oblige la cour à faire de même. Après le décès de son époux Henri II (I559) et de huit de ses enfants, Catherine de Médicis ne s'habille plus que d'un voile éternel (73). Michel Pastoureau souligne que dans de nombreux ordres monastiques et religieux, les clercs, les universitaires se vêtent d'un noir vertueux et austère. Selon lui, cette mode est due au progrès technique ayant permis de teindre des étoffes dans un noir profond vers I500 (7I). Le noir prend également son essor parmi le patriciat fortuné et perdure jusqu'au milieu du XVII ${ }^{\text {ème }}$ siècle.

Cette mode est à relier à l'iconographie macabre et surtout à la ritualisation du deuil lorsque l'habit noir envahit la scène funéraire (Ariès 88, I00-02). En I493, peu avant sa mort, Philippe Pot, grand Sénéchal de Bourgogne ${ }^{4}$ commande son propre monument funéraire. Le tombeau devait immortaliser sa cérémonie funéraire ${ }^{5}$ : huit pleurants à taille humaine supportent la dalle funéraire. Revêtus du costume de deuil, un grand manteau de drap noir à capuchon, ils portent les blasons où s'affichent les huit quartiers de noblesse du sénéchal, dont le blason de Palamède, chevalier de la Table Ronde. Le gisant de Philippe Pot est revêtu d'une armure et d'un tabard richement armorié ; il est représenté en tant que chevalier de la Toison d'or.

Ici, « la mort nue » de la dépouille mortelle des gisants squelettiques a fait place à une représentation du mort dans la fonction symbolique qu'il exerçait dans sa vie. Ce simulacre le montre tel qu'il veut perdurer dans l'imaginaire social et politique. Ce glissement de la réalité crue à la représentation fastueuse est plus évident encore au moment des funérailles. Ici les choses sont inversées, la représentation du gisant sous la forme de transit est en réalité très rare, alors que la représentation du

\footnotetext{
Il servit les ducs de Bourgogne, Philippe III le Bon, dont il était le filleul, puis son fils Charles le Téméraire lui servit de chambellan, il le nomma gouverneur de Bourgogne et le fit chevalier de l'ordre de Saint-Michel. Il sut conserver les faveurs de son successeur Charles VIII.

5. Vers I480-I483, provenant de la chapelle Saint-Jean-Baptiste de l'abbaye de Cîteaux (Côte-d'Or). Voir Sophie Jugie.
} 
corps social est, de loin, la plus fréquente. Le «théâtre de la mort », avec d'immenses tentures noires, squelettes, crânes etc., célébrait non seulement la mort mais surtout une identité sociale. Le décor des rites des obsèques comprend le nombre de jours de deuil, la pompe des cérémonies, le nombre et la taille même des cierges, traduisant le rang de la personne (Léonie 43). Quant aux armoiries, elles s'inscrivent dans la temporalité d'une lignée familiale (Aubert et Beaulieu 24I-45) que l'individu ne fait que perpétuer tout au long de sa vie pour passer le flambeau à la génération suivante. Les armoiries rappellent la coutume romaine, où un cortège avec les portraits des ancêtres accompagnait le défunt. Toutefois, ici, la personne n'est plus représentée, mais signifiée à travers son blason. Il est étonnant de constater qu'au moment où le portrait doté d'une présence réelle apparaît, comme celui de Jean II, dit le $B_{o n}{ }^{6}$, apparaît également cette autre face codifiée des armoiries, comme l'imago se substituant à la personne. Dès le début du XIII ${ }^{\text {ìme }}$ siècle, les verrières de la cathédrale de Chartres attestent des armoiries dans les églises.

\section{L'essor des armoiries}

La Chanson de Roland décrit les armes comme signes de reconnaissance : « Escuz unt genz, de multes cunoissances ... et leurs écus bien ouvrés sont parés de maintes connaissances » (chant CCXXV, vers $3090 ; 257)$.

Car c'est dès le $\mathrm{XI}^{\text {ème }}$ siècle, que les armoiries distinguent la noblesse et la royauté selon des règles déterminées. Mais c'est surtout avec la première croisade que les blasons prennent leur essor. Les signes héraldiques peints sur les armes, c'est-à-dire sur le bouclier, l'écu, la cotte d'armes, sont des signes de reconnaissance, de ralliement afin de rassembler des troupes de nationalités différentes et de distinguer leurs chevaliers sur les champs de bataille. Les tournois avec des boucliers pourvus de leurs armoiries sont un autre exemple du rite de la chevalerie (Pastoureau 1993, 407).

Une des premières mentions d'armoiries devant un fond noir est donnée par Olivier de la Marche lorsqu'il témoigne de la réunion annuelle des chevaliers de la Toison d'or en I445 à Gand (Bureaux). Les armoiries avec le nom et la devise du chevalier ont été placées dans le chœur de l'église SaintJean (aujourd'hui Saint-Bavon). Lorsqu'il interrogeait sur la raison des places vides occupées par des armoiries placées devant un tissu noir, on lui répondait que celles-ci remplaçaient la personne décédée.

Anonyme de l'Ecole Française, portrait de Jean II, dit le Bon, I355 conservé au Musée du Louvre. Voir la thèse de Martine Lacas, Construire la ressemblance : l'architecture du portrait à la Renaissance (2006). 
En même temps fleurit la présence des signes héraldiques, ce langage codifié et abstrait désigne d'abord une descendance royale, une parenté. Au XIV ${ }^{\text {ème }}$ siècle, cette logique d'appartenance s'élargit et prend une dimension sociale et politique en rattachant l'individu à une ville, une confrérie, une corporation et donc à un métier.

\section{Le Carême}

Si la litre et les blasons reflètent les liens sociaux et économiques d'une personne, ils rappellent originalement la liturgie du Carême. Le Rationale divinorum officiorum, un des écrits religieux les plus importants du Moyen Âge, stipule que soient voilés toutes les images, les crucifix, les reliques et les tabernacles dans la maison de Dieu pendant la période du Carême. Ainsi Philippe le Hardi, duc de Bourgogne, fit tendre durant deux caresme cothidianes, des draps gris et sombres, couleur de cendre. Citons l'évangile de Jean : «Pendant que vous avez la lumière, croyez en la lumière, afin que vous soyez des enfants de lumière. Jésus dit ces choses, puis il s'en alla, et se cacha loin d'eux » (Jean I2:36). En un temps où les croix, qu'elles fussent ou non reliquaires, étaient en métal précieux et incrustées de pierreries, un voile était suspendu entre le chœur et la nef dans les églises de façon à cacher complètement le sanctuaire, tant pour en atténuer l'éclat en ces jours de pénitence que pour le découvrir solennellement et le révéler au Vendredi saint. Même si ce rite fut adopté à la fois dans la liturgie papale au Sessonianum et dans celle des chanoines du Latran, ce n'est qu'à partir de I488 qu'on voile toutes les croix à Rome (Cabro 3003). Le drap du Carême nommé aussi drap de la faim rappelle d'abord le temps de la pénitence. Les images voilées parlent de la Passion et de la remémoration du Christ, sa descente aux limbes, de cet autre monde jusqu'au jour de sa résurrection. La mort est ici associée au noir du royaume des ténèbres et au silence.

Ce rite du voilement et du dévoilement se retrouve dans un registre plus individuel. La mort d'une personne exige de voiler la face des images et leurs supports ; les portraits et les miroirs de la maison seront recouverts d'un voile noir en signe de deuil. Le défunt, la vue éteinte, impose aux proches de se détourner des images et de se vêtir de noir.

Dans l'espace public, le « droit de litre » accorde au seigneur de l'église le privilège de s'approprier l'espace sacré et de faire porter le deuil à toute la communauté, au même titre que le droit de banc alors que l'homme simple fait silence dans sa maison. La litre du seigneur fait porter son deuil à l'église ou à la chapelle de sa paroisse. Ce privilège rappelle une autre coutume, celle d'avoir sa dépouille enfouie sous les dalles de la nef, garantissant au défunt une intercession privilégiée auprès des saints et surtout sa pérennité parmi les vivants. 
Il est important de situer la litre dans un contexte pictural où plusieurs paramètres entrent en jeu. L'avènement de la perspective linéaire vers le XIV ${ }^{\text {ème }}$ siècle conduit à une unification et à une hiérarchisation de l'espace pictural qui converge vers une représentation tridimensionnelle de l'espace et une représentation réaliste des protagonistes. Les couleurs sont également soumises à une logique de lumière et d'ombre, de blanc et de noir. La radicalité de la litre avec sa bande noire traversant l'espace blanc de l'église est d'expression quasiment abstraite. Au sens de Panofsky c'est une forme symbolique, qui révèle une nouvelle relation entre le sujet et le monde.

Elle annule les décors picturaux. La barre horizontale de la litre évoque les portraits d'Hans Holbein le Jeune. Une inscription en latin précisant le nom, l'âge de la personne peinte et la date de la réalisation du tableau, traverse la figure au niveau du visage. Elle brise l'illusion picturale. En outre, la verticalité du corps et l'horizontalité de l'inscription forment une croix. Deux conceptions de l'espace s'opposent ici : l'inscription renvoie au caractère commémoratif et au temps, tandis que la présence physique du portrait semble capter une personne vivante (Zwingenberger 53-67). Le trait horizontal est le signe pictural d'un symptôme culturel ou plus précisément d'une mentalité collective : Ici, la vie et la mort sont deux faces d'une même médaille.

Dans notre analyse, nous avons souligné que la couleur noire de la litre s’inscrit dans un contexte de deuil. La litre souligne sa valeur spatiale, car le pourtour noir sur les murs de l'église cerne l'espace sacré. Dans la liturgie du Carême, le drap de la faim recouvre les images sacrées pendant le temps de la pénitence. Lorsque le Christ ou le défunt entrent dans l'invisible, le visible disparaît. Il est intéressant également de placer l'avènement de la litre dans le contexte de l'histoire de l'art en Europe. Dès les débuts de la Renaissance, la ressemblance se construit dans le portrait et le paysage se creuse dans la perspective d'imiter la réalité. Au même moment apparaît la litre, cette ligne qui annule tout, en tirant un trait sur le visible ${ }^{7}$.

Dans la tradition de la litre, seules les armoiries seigneuriales subsistent par la représentation symbolique. La représentation de la personne et parfois le décor sacré dont il a été le commanditaire disparaissent après le temps du deuil. Les litres sont donc essentiellement éphémères. Elles étaient souvent recouvertes d'un badigeon blanc. Celui-ci recouvrait le décor pictural pour laisser place à un autre décor dont témoignent les couches successives.

La litre en tant qu'image survivante personnifie le défunt dans son rayonnement spirituel et social dans sa collectivité. Cette contraction du réel de la Vanitas se situe au-delà

Merci à Anouk Jevtik, restauratrice de peintures murales à Paris, pour son aide sur ce sujet bien complexe, plus connu des restaurateurs que des historiens d'art. 
de toute représentation. Le noir suscite un état d'âme tandis que le blason affiche sa ligne familiale. Mais surtout, s'expriment ici une pensée et une manifestation spatiale qui inaugurent en quelque sorte 1'ultime trait de l'art abstrait. La matière noire de la litre indique l'irreprésentable. $\mathrm{Au} \mathrm{XX}^{\text {ème }}$ siècle, Yves Klein l'exprime par l'espace du vide signifié par le dais de deuil sur la galerie Iris Clert, de même le carré noir sur le corbillard de Kasimir Malévitch. Aujourd'hui l'abstraction radicale de Pierre Soulages célèbre l'espace méditatif du noir-lumière ou outrenoir.

\section{OUVRaGes Cités}

ANDRE, Michel. Cours alphabétique, théorique et pratique de la législation civile ecclésiastique. Vol. 2. Paris : Bureau de la Voix de l'Église, I848.

ARIES, Philippe. Essais sur l'histoire de la mort en Occident. Paris : Le Seuil, 1975.

AUBERT, Michel et Michèle BEAULIEU. Description raisonnée des sculptures du Musée du Louvre. T. 1 : Moyen Age. Paris : Musées nationaux, 1950.

La Chanson de Roland. Trad. par Joseph Bédier. Paris : Henri Piazza, I923.

BODIN, Pierre. «Les litres funéraires »., Dixième Congrès International sur les Danses Macabres et l'art macabre en général (Vendôme, Loir-et-Cher, du 6 au Io septembre 2000).Meslay-le-Grenet, édité par l'Association Danses macabres d'Europe: 238-248.

BLONDAUX, Laurence. "Quelques exemples de litres funéraires », Onzième Congrès International d'études sur les danses macabres et l'art macabre en général, Rouen, du $\mathrm{I}^{\mathrm{er}}$ au 5 octobre 2003). Meslay-le-Grenet : Edité par l'Association Danses macabres d'Europe : 3I8-324.

Groupe de Recherches sur les Peintures Murales (BLONDAUX Laurence, CAFFIN Marie-Gabrielle, CZERNIAK Virginie, DAVY Christian, DECOTTIGNIES Sylvie, HANS-COLLAS Ilona, JUHEL Vincent, LEDUC Christine). Vifs nous sommes... morts nous serons. La Rencontre des trois morts et des trois vifs dans la peinture murale en France. Vendôme : Éditions du Cherche-Lune, 200I.

BUREAUX, Guillaume. Union et désunion de la noblesse en parade. Le rôle des Pas d'armes dans l'entretien des rivalités chevaleresques entre cours princières occidentales, XVe-XVIe siècles (Anjou, Bourgogne, France, Saint-Empire). Rouen : Édition Université de Rouen Normandie, 2018.

CABRO, Dom Fernand. Dictionnaire d'Archéologie Chrétienne et de Liturgie. Paris : Librairie Letouzey et Ane, I9I4.

DAVY, Christian et Laurence BLONDAUX. «À propos des armoiries de Bouchamps-lès-Craon, quelques précisions sur la litre seigneuriale ». Murs... Murs, Bulletin d'information du Groupe de Recherches sur la Peinture Murale 53 (2018) : 3-I3. 
ERNOUT, Alfred et Antoine MEILLET. Dictionnaire étymologique de la langue latine. Histoire des mots. Paris : Édition Klincksieck, 1959.

HEGEL Georg Wilhelm Friedrich. Phénoménologie de l'esprit. Préface et trad. par Jean Hyppolite. Paris : Édition Aubier, 1999, t. I.

HORACE, Épitres I. Trad. par F. Villeneuve. Paris : Édition Les Belles Lettres, 1989.

JUGIE, Sophie. Le Tombeau de Philippe Pot. Paris : Musée du Louvre ; Ediciones El Viso, 2018.

LACAS, Martine. Construire la ressemblance : l'architecture du portrait à la Renaissance. Dir. Yves Hersant. Paris : Édition EHESS, 2006.

LEONIE, Sylviane. «Le Théâtre de la mort ». Le Régent : entre fable et histoire. Dir. Denis Reynaud et Chantal Thomas. Paris : Édition CNRS, 2003. 42-62.

MENESTRIER, Claude-François. Des décorations funèbres. Paris : J.-B. de la Caille, I684.

PASTOUREAU, Michel. Noir. Histoire d'une couleur. Paris : Édition Points, 2014.

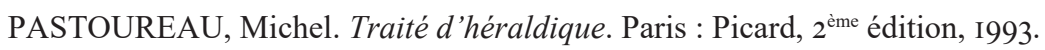

RAVIEZ, François. «Une Ligne de larmes ou Madame de Saint-Simon au miroir des Mémoires » Le Couple au XVIII İme siècle : Réalités et représentations, textes réunis par Marie-Odile BERNEZ, Dijon : EUD, 200I. 43-49

TESSIER, André. « Le genre décoratif funèbre ». Revue de l'art ancien et moderne XLVI (1924) : 274-282.

ZWINGENBERGER, Jeanette. Hans Holbein le Jeune. L'ombre de la mort. Londres : Édition Parkstone, I999. 